\title{
Exact Pairwise Error Probability of Differential Space-Time Codes in Spatially Correlated Channels
}

\author{
Tharaka A. Lamahewa ${ }^{\dagger}$, Van K. Nguyen ${ }^{\S}$, Thushara D. Abhayapala ${ }^{\dagger}$ \\ $\dagger$ Department of Information Engineering, The Australian National University, Canberra ACT 0200, Australia.

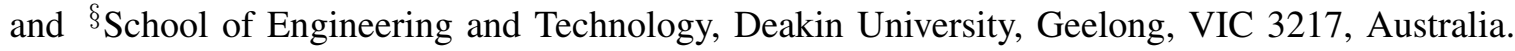 \\ \{tharaka.lamahewa, thushara.abhayapala\}@anu.edu.au, vknguyen@deakin.edu.au.
}

\begin{abstract}
In this paper, we derive an analytical expression for the exact pairwise error probability (PEP) of a differential space-time coded system operating over a spatially correlated slow fading channel. An analytic model for spatial correlation is used which fully accounts for antenna spacing, antenna geometry and non-isotropic scattering distributions. Inclusion of spatial information in error performance analysis provides valuable insights into the physical factors determining the performance of a differential space-time code (DSTC). Using this new PEP expression, we investigate the effects of antenna spacing, antenna geometries and azimuth power distribution parameters (angle of arrival/departure and angular spread) on the performance of a differential space-time block code (DSTBC) proposed in the literature for two transmit antennas.
\end{abstract}

\section{INTRODUCTION}

Multiple-input multiple-output (MIMO) communications systems using multi-antenna arrays simultaneously during transmission and reception have generated significant interest in recent years. Under the assumption that the fading channel coefficients between different antenna elements are statistically independent and known at the receiver (coherent detection), theoretical work of [1] and [2] revealed that the channel capacity of multiple-antenna array communication systems scales linearly with the smaller of the number of transmit and receive antennas. Motivated by these works, [3-5] have proposed several modulation and coding schemes, namely space-time trellis codes and space-time block codes, to exploit the potential increase in capacity and diversity gain using multi antenna arrays with coherent detection. The effectiveness of these schemes heavily relies on the accuracy of the channel estimation at the receiver. Therefore, differential space-time coding schemes make an attractive alternative to combat inaccuracy of channel estimation in above schemes. With differential space-time coding schemes channel state information is not required at either end of the channel. Several differential space-time coding schemes for multi-antenna systems have been proposed in [6-8] and the error performance of some of these schemes have been investigated in [9-11]. In [9], a closed form expression of bit error probability of DSTBCs

This work was supported by the Australian Research Council Discovery Grant DP0343804. T. D. Abhayapala is also with National ICT Australia, Locked Bag 8001, Canberra, ACT 2601, Australia. National ICT Australia is funded through the Australian Government's Backing Australia's Ability initiative, in part through the Australian Research Council. based on Alamouti's scheme was derived assuming fading channels are statistically independent. By applying the theory of Gaussian quadratic forms, an upper bound for the PEP of DSTCs was derived in [10] for arbitrary correlated channels. Following a similar approach, [11] has derived a closed form expression of the exact pairwise error probability (exact-PEP) at asymptotically high signal-to-noise ratios (SNR) of the DSTC in spatially correlated fading channels.

This paper presents analytical expressions developed to analyze the error performance of a multi-antenna system employing DSTC under spatially correlated fading channels. In particular, using the moment generating function (MGF) based approach presented in [12] and [13], this paper presents an alternative expression for the exact-PEP of a differential space-time coding scheme at asymptotically high SNR operating over a spatially correlated slow fading channel. Also the PEP upper bound of the DSTC in spatially correlated channels is derived from the exact-PEP expression. Compared with the asymptotic PEP expression in [11], this new expression is much closer to the true PEP at low SNRs. Furthermore, this expression fully accounts for antenna spacing and antenna geometry (Uniform Linear Array (ULA), Uniform Grid Array (UGA), Uniform Circular Array (UCA), etc.) along with non-isotropic scattering distributions (Uniform, Gaussian, Laplacian, Von-mises, etc.) surrounding the transmit and receive antenna arrays. For uncorrelated channels, a closed form expression for the exact-PEP of DSTC is derived, and for correlated channels, an analytical technique is given which can be used to evaluate the exact-PEP of DSTC in closed form. The strength of our new analytical PEP expression is demonstrated by evaluating the performance of a DSTBC with two transmit antennas proposed by Tarokh et al. [6].

Notations: Throughout the paper, the following notations will be used: $[\cdot]^{T},[\cdot]^{*}$ and $[\cdot]^{\dagger}$ denote the transpose, complex conjugate and conjugate transpose operations, respectively. The symbols $\delta(\cdot)$ and $\otimes$ denote the Dirac delta function and Matrix Kronecker product, respectively. The notation $E\{\cdot\}$ denotes the mathematical expectation, $\mathrm{Q}(y)=\int_{-\infty}^{y} e^{-x^{2} / 2} d x$ denotes the Gaussian Q-function, $\|\cdot\|$ denotes the Euclidean norm of a vector, $|\boldsymbol{A}|$ denotes the determinant of matrix $\boldsymbol{A}$, $\operatorname{vec}(\boldsymbol{A})$ denotes the vectorization operator which stacks the 
columns of $\boldsymbol{A}$, and $\lceil$.$\rceil denotes the ceiling operator. The matrix$ $\boldsymbol{I}_{n}$ is the $n \times n$ identity matrix.

\section{SySTEM MODEL}

Consider a MIMO system consisting of $n_{T}$ transmit antennas and $n_{R}$ receive antennas. Let $\boldsymbol{X}_{k}$ be the $k$-th $n_{T} \times T$ code matrix to be transmitted by $n_{T}$ transmit antennas over $T$ symbol intervals. At the start of the transmission, the transmitter sends the code matrix $\boldsymbol{X}_{0}=\boldsymbol{D}$. Thereafter, information is differentially encoded according to the rule

$$
\boldsymbol{X}_{k}=\boldsymbol{X}_{k-1} \boldsymbol{S}_{\ell(k)}, \text { for } k=1,2, \cdots
$$

where $\boldsymbol{S}_{\ell(k)} \in \mathbb{C}^{n_{T} \times T}$ is the $k$-th information matrix which is an element of a group of unitary space-time modulated constellation matrices $\mathcal{V}$ of size $L$ with unitary property $\boldsymbol{S}_{\ell(k)} \boldsymbol{S}_{\ell(k)}^{\dagger}=$ $\boldsymbol{I}$ for $\ell(k)=0,1, \cdots, L-1$ [7]. This unitary space-time constellation can be constructed based on orthogonal designs [5] or group designs [7,8]. Similar to [7,8] we assume that $T=n_{T}$ and also $\boldsymbol{D}=\boldsymbol{I}_{n_{T}}$. As a result, $\boldsymbol{X}_{k}$ is also unitary.

Let $\boldsymbol{H} \in \mathbb{C}^{n_{R} \times n_{T}}$ be the unknown fading channel gain matrix and $\boldsymbol{N}_{k} \in \mathbb{C}^{n_{R} \times n_{T}}$ be the additive noise matrix, then the received signal $\boldsymbol{Y}_{k} \in \mathbb{C}^{n_{R} \times n_{T}}$ corresponding to the $k$-th space-time codeword $\boldsymbol{X}_{k}$ can be written as

$$
\boldsymbol{Y}_{k}=\sqrt{E_{s}} \boldsymbol{H} \boldsymbol{X}_{k}+\boldsymbol{N}_{k}, \text { for } k=0,1,2, \cdots
$$

where $E_{s}$ is the average transmitted signal energy per symbol period. Each of the elements of $\boldsymbol{N}_{k}$ is assumed to be independently and identically distributed zero-mean complex Gaussian random variable with variance $\sigma_{n}^{2} / 2$ per complex dimension. The $(p, q)$ th entry of $\boldsymbol{H}$ is the complex channel fading gain from transmit antenna $q$ to receive antenna $p$ and fading gains are assumed to be flat Rayleigh fading.

At the receiver, the transmitted signal can be noncoherently demodulated by using two consecutive observations, $\boldsymbol{Y}_{k-1}$ and $\boldsymbol{Y}_{k}$. We assume that the channel matrix $\boldsymbol{H}$ remains constant for $\boldsymbol{Y}_{k-1}$ and $\boldsymbol{Y}_{k}$. Signals $\boldsymbol{Y}_{k-1}$ and $\boldsymbol{Y}_{k}$ can be expressed in vector form (row) as

$$
\begin{aligned}
\boldsymbol{y}_{k-1} & =\sqrt{E_{s}} \boldsymbol{h} \mathcal{X}_{k-1}+\boldsymbol{n}_{k-1} \\
\boldsymbol{y}_{k} & =\sqrt{E_{s}} \boldsymbol{h} \mathcal{X}_{k}+\boldsymbol{n}_{k} \\
& =\boldsymbol{y}_{k-1} \mathcal{S}_{\ell(k)}+\boldsymbol{w}_{k}
\end{aligned}
$$

where $\boldsymbol{y}_{k}=\operatorname{vec}\left(\boldsymbol{Y}_{k}^{T}\right)^{T}, \boldsymbol{\mathcal { X }}_{k}=\boldsymbol{I}_{n_{R}} \otimes \boldsymbol{X}_{k}, \boldsymbol{h}=\operatorname{vec}\left(\boldsymbol{H}^{T}\right)^{T}$, $\boldsymbol{n}_{k}=\operatorname{vec}\left(\boldsymbol{N}_{k}^{T}\right)^{T}, \mathcal{S}_{\ell(k)}=\boldsymbol{I}_{n_{R}} \otimes \boldsymbol{S}_{\ell(k)}$ and $\boldsymbol{w}_{k}=\boldsymbol{n}_{k}-$ $\boldsymbol{n}_{k-1} \mathcal{S}_{\ell(k)}$. To obtain $\boldsymbol{y}_{k}$ and $\boldsymbol{y}_{k-1}$, we have used the $\operatorname{vec}(\cdot)$ identity $\operatorname{vec}(\boldsymbol{A} \boldsymbol{X} \boldsymbol{B})=\left(\boldsymbol{B}^{T} \otimes \boldsymbol{A}\right) \operatorname{vec}(\boldsymbol{X})$. From (4), the transmitted data matrix is differentially detected using the following maximum likelihood receiver

$$
\begin{aligned}
\widehat{\boldsymbol{S}} & =\arg \min _{\boldsymbol{S} \in \mathcal{V}}\left\|\boldsymbol{y}_{k}-\boldsymbol{y}_{k-1} \mathcal{S}\right\|^{2} \\
& =\arg \max _{\boldsymbol{S} \in \mathcal{V}} \operatorname{Re}\left\{\boldsymbol{y}_{k-1} \boldsymbol{S} \boldsymbol{y}_{k}^{\dagger}\right\}
\end{aligned}
$$

\section{A. Spatial Channel Model and Channel Correlation}

Using a recently developed 2-dimensional spatial channel model $^{1}$ [14], we are able to incorporate the antenna spacing, antenna placement (ULA, UCA, UGA, etc.) and scattering distribution parameters such as mean angle-of-arrival (AOA), mean angle-of-departure (AOD) and angular spread, into the exact-PEP calculations of differential space-time coded systems. In this model, the MIMO channel $\boldsymbol{H}$ is decomposed into deterministic and random parts as

$$
\boldsymbol{H}=\boldsymbol{J}_{R} \boldsymbol{H}_{S} \boldsymbol{J}_{T}^{\dagger},
$$

where $\boldsymbol{J}_{T}$ is the $n_{T} \times\left(2 m_{T}+1\right)$ transmit antenna array configuration matrix and $\boldsymbol{J}_{R}$ is the $n_{R} \times\left(2 m_{R}+1\right)$ receive antenna array configuration matrix, where $\left(2 m_{T}+1\right)$ and $\left(2 m_{R}+1\right)$ are the number of effective communication modes ${ }^{2}$ available in the transmitter and receiver regions, respectively. Note that, $m_{T}$ and $m_{R}$ are determined by the size of the antenna aperture, but not from the number of antennas encompassed in an antenna array. The number of effective communication modes $(M)$ available at a region is given by [15]

$$
M \triangleq 2\lceil\pi e r / \lambda\rceil+1
$$

where $r$ is the minimum radius of the antenna array aperture, $\lambda$ is the wavelength and $e \approx 2.7183$. We refer the reader to [14] for the definitions of $\boldsymbol{J}_{R}$ and $\boldsymbol{J}_{T}$. Finally, $\boldsymbol{H}_{S}$ is the $\left(2 m_{R}+1\right) \times\left(2 m_{T}+1\right)$ random scattering matrix with $(n, m)$ th element given by

$$
\begin{aligned}
\left\{\boldsymbol{H}_{S}\right\}_{n, m} & =\int_{0}^{\pi} \int_{0}^{\pi} g(\varphi, \phi) e^{-i\left(n-m_{R}-1\right) \phi} e^{i\left(m-m_{T}-1\right) \varphi} d \phi d \varphi \\
n & =1, \cdots, 2 m_{R}+1, \quad m=1, \cdots, 2 m_{T}+1 .
\end{aligned}
$$

Note that $\left\{\boldsymbol{H}_{S}\right\}_{n, m}$ represents the complex gain of the scattering channel between the $m$-th mode of the transmitter region and the $n$-th mode of the receiver region, where $g(\varphi, \phi)$ is the scattering gain function, which is the effective random complex gain for signals leaving the transmitter aperture with angle of departure $\varphi$ and arriving at the receiver aperture with angle of arrival $\phi$.

The correlation matrix of the channel $\boldsymbol{H}$ can be written as

$$
\boldsymbol{R}=E\left\{\boldsymbol{h}^{\dagger} \boldsymbol{h}\right\}=\left(\boldsymbol{J}_{R}^{*} \otimes \boldsymbol{J}_{T}\right) \boldsymbol{R}_{S}\left(\boldsymbol{J}_{R}^{T} \otimes \boldsymbol{J}_{T}^{\dagger}\right),
$$

where $\boldsymbol{h}=\left(\operatorname{vec}\left(\boldsymbol{H}^{T}\right)\right)^{T}$ and $\boldsymbol{R}_{S}$ the correlation matrix of the scattering channel, which is defined as $\boldsymbol{R}_{S}=E\left\{\boldsymbol{h}_{S}^{\dagger} \boldsymbol{h}_{S}\right\}$ with $\boldsymbol{h}_{S}=\left(\operatorname{vec}\left(\boldsymbol{H}_{S}^{T}\right)\right)^{T}$.

In some circumstances, $\boldsymbol{R}_{S}$ can be expressed as a Kronecker product between modal correlation matrices observed at the transmitter and the receiver antenna arrays [16], i.e.,

$$
\boldsymbol{R}_{S}=E\left\{\boldsymbol{h}_{S}^{\dagger} \boldsymbol{h}_{S}\right\}=\boldsymbol{F}_{R} \otimes \boldsymbol{F}_{T},
$$

here $\boldsymbol{F}_{R}$ and $\boldsymbol{F}_{T}$ are the receive and transmit modal correlation matrices. Substituting (10) in (9) gives

$$
\boldsymbol{R}=\left(\boldsymbol{J}_{R}^{*} \boldsymbol{F}_{R} \boldsymbol{J}_{R}^{T}\right) \otimes\left(\boldsymbol{J}_{T} \boldsymbol{F}_{T} \boldsymbol{J}_{T}^{\dagger}\right)
$$

\footnotetext{
${ }^{1}$ The 2-D case is a special case of the 3-D case where all the signals arrive from on a horizontal plane only.

${ }^{2}$ The set of modes form a basis of functions for representing a multipath wave field.
} 


\section{EXACT PEP of DifFEREnTIAL SPACE-Time Codes}

Based on (5), the receiver will erroneously select $\boldsymbol{S}_{j}$ when $\boldsymbol{S}_{i}$ was actually sent as the $k$-th information matrix if

$$
\begin{aligned}
\left\|\boldsymbol{y}_{k}-\boldsymbol{y}_{k-1} \mathcal{S}_{j}\right\|^{2} & \leq\left\|\boldsymbol{y}_{k}-\boldsymbol{y}_{k-1} \mathcal{S}_{i}\right\|^{2} \\
\boldsymbol{y}_{k-1} \boldsymbol{D}_{i, j} \boldsymbol{y}_{k-1}^{\dagger} & \leq 2 \operatorname{Re}\left\{\boldsymbol{w}_{k} \boldsymbol{\Delta}_{i, j}^{\dagger} \boldsymbol{y}_{k-1}^{\dagger}\right\}
\end{aligned}
$$

where $\boldsymbol{\Delta}_{i, j}=\mathcal{S}_{j}-\mathcal{S}_{i}=\boldsymbol{I}_{n_{R}} \otimes\left(\boldsymbol{S}_{j}-\boldsymbol{S}_{i}\right)$ and $\boldsymbol{D}_{i, j}=$ $\boldsymbol{\Delta}_{i, j} \boldsymbol{\Delta}_{i, j}^{\dagger}=\boldsymbol{I}_{n_{R}} \otimes\left(\left(\boldsymbol{S}_{i}-\boldsymbol{S}_{j}\right)\left(\boldsymbol{S}_{i}-\boldsymbol{S}_{j}\right)^{\dagger}\right)$ is the code distance matrix. For given $\boldsymbol{y}_{k-1}$, the term on the left hand side of (12b) is a constant and the term on the right hand side is a Gaussian random variable.

Let $u=2 \operatorname{Re}\left\{\boldsymbol{w}_{k} \boldsymbol{\Delta}_{i, j}^{\dagger} \boldsymbol{y}_{k-1}^{\dagger}\right\}$, then it can be shown ${ }^{3}$ that $u$ has the conditional mean

$$
\begin{aligned}
\bar{m} & =E\left\{u \mid \boldsymbol{y}_{k-1}\right\}, \\
& =2 \operatorname{Re}\left\{\bar{m}_{\boldsymbol{n}_{k-1} \mid \boldsymbol{y}_{k-1}}\left(\boldsymbol{I}-\mathcal{S}_{i} \mathcal{S}_{j}^{\dagger}\right) \boldsymbol{y}_{k-1}^{\dagger}\right\}
\end{aligned}
$$

where $\bar{m}_{\boldsymbol{n}_{k-1} \mid \boldsymbol{y}_{k-1}}=\sigma_{n}^{2} \boldsymbol{y}_{k-1}\left(\boldsymbol{\mathcal { X }}_{k-1}^{\dagger} \boldsymbol{R} \mathcal{X}_{k-1}+\sigma_{n}^{2} \boldsymbol{I}_{n_{T} n_{R}}\right)^{-1}$, and the conditional variance

$$
\begin{aligned}
\sigma^{2} & =E\left\{\|u-\bar{m}\|^{2} \mid \boldsymbol{y}_{k-1}\right\} \\
& =2 \boldsymbol{y}_{k-1} \boldsymbol{\Delta}_{i, j}\left(\sigma_{n}^{2} \boldsymbol{I}+\mathcal{S}_{i}^{\dagger} \Sigma_{\boldsymbol{n}_{k-1} \mid \boldsymbol{y}_{k-1}} \mathcal{S}_{i}\right) \boldsymbol{\Delta}_{i, j}^{\dagger} \boldsymbol{y}_{k-1}^{\dagger},
\end{aligned}
$$

where $\Sigma_{\boldsymbol{n}_{k-1} \mid \boldsymbol{y}_{k-1}}=\sigma_{n}^{2}\left(\boldsymbol{I}-\sigma_{n}^{2}\left(E_{s} \mathcal{X}_{k-1}^{\dagger} \boldsymbol{R} \mathcal{X}_{k-1}+\sigma_{n}^{2} \boldsymbol{I}\right)^{-1}\right)$.

Let $d_{i, j}^{2}=\boldsymbol{y}_{k-1} \boldsymbol{D}_{i, j} \boldsymbol{y}_{k-1}^{\dagger}$, the PEP is then given by

$$
\begin{aligned}
\mathrm{P}\left(\boldsymbol{S}_{i} \rightarrow \boldsymbol{S}_{j} \mid \boldsymbol{y}_{k-1}\right) & =\operatorname{Pr}\left(u>d_{i, j}^{2}\right), \\
& =\int_{d_{i, j}^{2}}^{\infty} \frac{1}{\sqrt{2 \pi} \sigma} \exp \left(-\frac{(u-\bar{m})^{2}}{2 \sigma^{2}}\right) \mathrm{d} u \\
& =\mathrm{Q}\left(\frac{d_{i, j}^{2}-\bar{m}}{\sigma}\right) .
\end{aligned}
$$

By using Craig's formula for the Gaussian Q-function [17, Chap. 4, Eq. (4.2)]

$$
\mathrm{Q}(x)=\frac{1}{\pi} \int_{0}^{\pi / 2} \exp \left(-\frac{x^{2}}{2 \sin ^{2} \theta}\right) \mathrm{d} \theta
$$

and the MGF-based technique presented in [12], we can write the average PEP as

$$
\begin{aligned}
\mathrm{P}\left(\boldsymbol{S}_{i} \rightarrow \boldsymbol{S}_{j}\right) & =\frac{1}{\pi} \int_{0}^{\pi / 2} \int_{0}^{\infty} \exp \left(-\frac{\Gamma}{2 \sin ^{2} \theta}\right) p_{\Gamma}(\Gamma) d \Gamma \mathrm{d} \theta \\
& =\frac{1}{\pi} \int_{0}^{\pi / 2} \mathcal{M}_{\Gamma}\left(-\frac{1}{2 \sin ^{2} \theta}\right) \mathrm{d} \theta
\end{aligned}
$$

where $\mathcal{M}_{\Gamma}(s) \triangleq \int_{0}^{\infty} e^{s \Gamma} p_{\Gamma}(\Gamma) d \Gamma$ is the MGF of

$$
\Gamma=\frac{\left(d_{i, j}^{2}-\bar{m}\right)^{2}}{\sigma^{2}}
$$

and $p_{\Gamma}(\Gamma)$ is the probability density function of $\Gamma$. Finding MGF of $\Gamma$ in (17) poses a much harder problem. However, at asymptotically high SNRs (i.e., keep $E_{s}$ constant and $\sigma_{n}^{2} \rightarrow 0$ ) the conditional mean and the conditional variance of $u$ reduce to $\bar{m}=0$ and $\sigma^{2}=4 \sigma_{n}^{2} d_{i, j}^{2}$, respectively, and $\Gamma$ reduces to

$$
\Gamma=\frac{1}{4 \sigma_{n}^{2}} \boldsymbol{y}_{k-1} \boldsymbol{D}_{i, j} \boldsymbol{y}_{k-1}^{\dagger} \text {. }
$$

${ }^{3}$ Proofs will be reported in a future publication.
In this case $\Gamma$ is a quadratic form of a random variable since $\boldsymbol{y}_{k-1}$ is zero-mean complex Gaussian distributed random vector with covariance $\boldsymbol{R}_{\boldsymbol{y}_{k-1}}=E_{s} \boldsymbol{\mathcal { X }}_{k-1}^{\dagger} \boldsymbol{R} \boldsymbol{\mathcal { X }}_{k-1}+\sigma_{n}^{2} \boldsymbol{I}$ and $\boldsymbol{D}_{i, j}$ is Hermitian and also fixed for given two code words. Note that $\boldsymbol{R}$ is the correlation matrix of the channel, defined by (11). The MGF associated with a quadratic random variable of form (18) is readily found in the literature [18]. Using [18, Eq. 14], the MGF of $\Gamma$ can be written as

$$
\mathcal{M}_{\Gamma}(s)=\left|\boldsymbol{I}-\frac{s}{4 \sigma_{n}^{2}} \boldsymbol{R}_{\boldsymbol{y}_{k-1}} \boldsymbol{D}_{i, j}\right|^{-1} .
$$

Recalling the definition of $\boldsymbol{R}_{y_{k-1}}$, we may write the MGF of $\Gamma$ as

$$
\mathcal{M}_{\Gamma}(s)=\left|\boldsymbol{I}-\frac{s}{4}\left(\bar{\gamma} \boldsymbol{\mathcal { X }}_{k-1}^{\dagger} \boldsymbol{R} \boldsymbol{\mathcal { X }}_{k-1}+\boldsymbol{I}\right) \boldsymbol{D}_{i, j}\right|^{-1},
$$

where $\bar{\gamma}=E_{s} / \sigma_{n}^{2}$ is the average symbol energy-to-noise ratio, then the exact-PEP of a DSTC operating over a spatially correlated MIMO channel $\boldsymbol{H}$ is given by

$$
\begin{aligned}
& \mathrm{P}\left(\boldsymbol{S}_{i} \rightarrow \boldsymbol{S}_{j}\right)= \\
& \quad \frac{1}{\pi} \int_{0}^{\pi / 2}\left|\boldsymbol{I}+\frac{1}{8 \sin ^{2} \theta}\left(\bar{\gamma} \mathcal{X}_{k-1}^{\dagger} \boldsymbol{R} \mathcal{X}_{k-1}+\boldsymbol{I}\right) \boldsymbol{D}_{i, j}\right|^{-1} \mathrm{~d} \theta,
\end{aligned}
$$

where $\boldsymbol{R}$ is the channel correlation matrix, defined by (11). Eq. (21) reveals that the error performance of differentially spacetime coded systems depends not only the channel correlation matrix $\boldsymbol{R}$ and the code distance matrix $\boldsymbol{D}_{i, j}$, but also on the previously transmitted code matrix $\boldsymbol{X}_{k-1}$.

Since the maximum of the integrand occurs at the upper limit, i.e., for $\theta=\pi / 2$, replacing the integrand by its maximum value immediately gives the Chernoff upper bound

$$
\mathrm{P}\left(\boldsymbol{S}_{i} \rightarrow \boldsymbol{S}_{j}\right) \leq \frac{1}{2} \frac{1}{\left|\boldsymbol{I}+\frac{1}{8}\left(\bar{\gamma} \mathcal{X}_{k-1}^{\dagger} \boldsymbol{R} \mathcal{X}_{k-1}+\boldsymbol{I}\right) \boldsymbol{D}_{i, j}\right|} .
$$

In this paper, we mainly focus on the space-time modulated constellations with the property

$$
\left(\boldsymbol{S}_{i}-\boldsymbol{S}_{j}\right)\left(\boldsymbol{S}_{i}-\boldsymbol{S}_{j}\right)^{\dagger}=\beta_{i, j} \boldsymbol{I}_{n_{T}}, \forall i \neq j,
$$

where $\beta_{i, j}$ is a scalar. Space-time orthogonal designs [5] and some cyclic and dicyclic space-time modulated constellations in [7] are some examples which satisfy property (23) above. Applying (23) on (21) and using the unitary property of $\boldsymbol{X}_{k-1}$ and the determinant identity $|\boldsymbol{I}+\boldsymbol{A} \boldsymbol{B}|=|\boldsymbol{I}+\boldsymbol{B} \boldsymbol{A}|$, after straight forward manipulations, we can simplify exact-PEP (21) to

$$
\mathrm{P}\left(\boldsymbol{S}_{i} \rightarrow \boldsymbol{S}_{j}\right)=\frac{1}{\pi} \int_{0}^{\pi / 2}\left|\boldsymbol{I}+\frac{\beta_{i, j}}{8 \sin ^{2} \theta}(\bar{\gamma} \boldsymbol{R}+\boldsymbol{I})\right|^{-1} \mathrm{~d} \theta
$$

and the Chernoff upper bound (22) to

$$
\mathrm{P}\left(\boldsymbol{S}_{i} \rightarrow \boldsymbol{S}_{j}\right) \leq \frac{1}{2} \frac{\left(\frac{8+\beta i, j}{8}\right)^{-n_{T} n_{R}}}{\left|\boldsymbol{I}+\frac{\beta_{i, j} \bar{\gamma}}{\left(8+\beta_{i, j}\right)} \boldsymbol{R}\right|}
$$

With the property (23), it now becomes evident that error performance of DSTC is independent of the previously transmitted code matrix $\boldsymbol{X}_{k-1}$. 


\section{A. Exact-PEP for Uncorrelated Channels}

When the fading channels are independent and identically distributed (i.e., $\boldsymbol{R}=\boldsymbol{I}$ ), (24) simplifies to,

$$
\begin{aligned}
\mathrm{P}\left(\boldsymbol{S}_{i} \rightarrow \boldsymbol{S}_{j}\right) & =\frac{1}{\pi} \int_{0}^{\pi / 2}\left(\boldsymbol{I}+\frac{\beta_{i, j}}{8 \sin ^{2} \theta}(\bar{\gamma}+1)\right)^{-n_{T} n_{R}} \mathrm{~d} \theta, \\
& =\frac{1}{\pi} \int_{0}^{\pi / 2}\left(\frac{\sin ^{2} \theta}{\sin ^{2} \theta+\eta}\right)^{n_{T} n_{R}} \mathrm{~d} \theta,
\end{aligned}
$$

where $\eta=\beta_{i, j}(1+\bar{\gamma}) / 8$. Using a result found in [17], integral (26) can be evaluated in closed form as

$$
\mathrm{P}\left(\boldsymbol{S}_{i} \rightarrow \boldsymbol{S}_{j}\right)=\frac{1}{2}\left\{1-\sqrt{\frac{\eta}{1+\eta}} \sum_{\ell=0}^{n_{T} n_{R}-1}\left(\begin{array}{c}
2 \ell \\
\ell
\end{array}\right) \frac{1}{4^{\ell}(1+\eta)^{\ell}}\right\} .
$$

This expression illustrates that the exact PEP of a differential space time code (DSTC) operating over an uncorrelated channel depends only on $\beta_{i, j}$, which relates to the code distance matrix $\left(\boldsymbol{S}_{i}-\boldsymbol{S}_{j}\right)\left(\boldsymbol{S}_{i}-\boldsymbol{S}_{j}\right)^{\dagger}$. In the next section, we discuss a technique which can be used to evaluate the exactPEP in closed form for correlated channels.

\section{B. Exact-PEP for Correlated Channels}

Let $\boldsymbol{Z}=\beta_{i, j}(\bar{\gamma} \boldsymbol{R}+\boldsymbol{I}) / 8$ in (24). Suppose matrix $\boldsymbol{Z}$ has $K$ non-zero eigenvalues, including multiplicity, $\lambda_{1}, \lambda_{2}, \cdots, \lambda_{K}$, and the decomposition $\boldsymbol{Z}=\boldsymbol{U} \boldsymbol{D} \boldsymbol{U}^{-1}$, where $\boldsymbol{U}$ is the matrix of eigenvectors of $\boldsymbol{Z}$ and $\boldsymbol{D}$ is a diagonal matrix with the eigenvalues of $\boldsymbol{Z}$ on the diagonal. Then (24) becomes

$$
\begin{aligned}
\mathrm{P}\left(\boldsymbol{S}_{i} \rightarrow \boldsymbol{S}_{j}\right) & =\frac{1}{\pi} \int_{0}^{\pi / 2}\left|\boldsymbol{I}+\frac{1}{\sin ^{2} \theta} \boldsymbol{Z}\right|^{-1} \mathrm{~d} \theta, \\
& =\frac{1}{\pi} \int_{0}^{\pi / 2}\left|\boldsymbol{I}+\frac{1}{\sin ^{2} \theta} \boldsymbol{D}\right|^{-1} \mathrm{~d} \theta, \\
& =\frac{1}{\pi} \int_{0}^{\pi / 2} \prod_{\ell=1}^{K}\left(\frac{\sin ^{2} \theta}{\lambda_{\ell}+\sin ^{2} \theta}\right)^{m_{\ell}} \mathrm{d} \theta,
\end{aligned}
$$

where $m_{\ell}$ is the multiplicity of eigenvalue $\lambda_{\ell}$. Using the partial fraction expansion technique given in [13] and a result found in [17], the integral in (28) can be evaluated in closed form.

Recall the definition of the channel correlation matrix $\boldsymbol{R}$ given in (9). When $\boldsymbol{R}_{S}=\boldsymbol{I}$ (i.e., correlation between different communication modes is zero), Eq. (28) above captures the effects due to antenna spacing and antenna geometry on the performance of a differentially space-time coded communications system.

\section{Analytical Performance Evaluation}

As an example, we consider the rate- $12 \times 2$ space-time modulated constellation set $\mathcal{V} \equiv\left\{\boldsymbol{S}_{i} \mid \boldsymbol{S}_{i} \boldsymbol{S}_{i}^{\dagger}=\boldsymbol{I}, i=0, \cdots, 3\right\}$, derived in [5] based on orthogonal designs with

$$
\boldsymbol{S}_{i}=\left[\begin{array}{cc}
s 1 & -s 2 \\
s 2 & s 1
\end{array}\right], \text { for } i=0, \cdots, 3,
$$

where $s_{i}, i=1,2$ are symbols drawn from the normalized BPSK alphabet $\{ \pm 1 / \sqrt{2}\}$. Let $\boldsymbol{S}_{0}$ and $\boldsymbol{S}_{1}$ correspond to the matrix with $\left(s_{1}, s_{2}\right)=(1 / \sqrt{2}, 1 / \sqrt{2})$ and $\left(s_{1}, s_{2}\right)=$

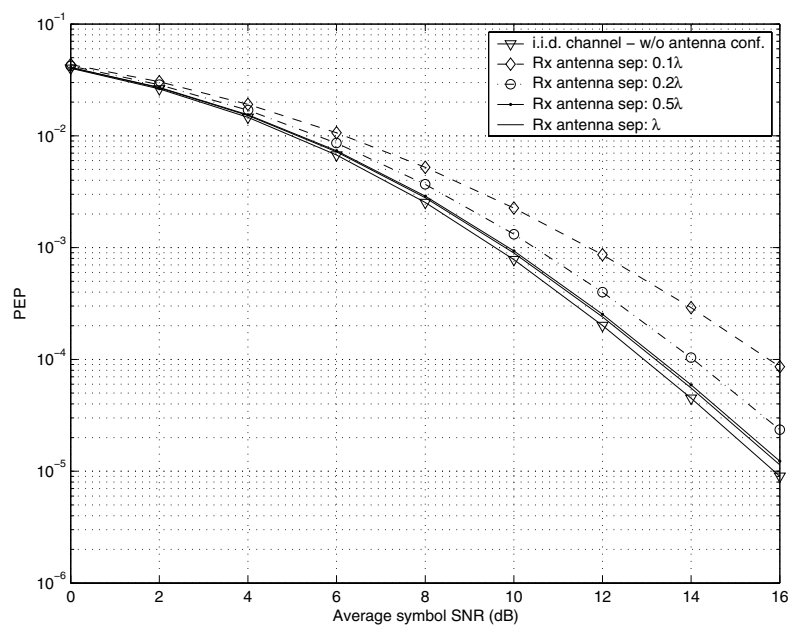

Fig. 1. Exact-PEP performance of DSTC scheme with two transmit and two receive antennas for transmit antenna separation $0.5 \lambda$ and $\beta_{0,1}=2$.

$(1 / \sqrt{2},-1 / \sqrt{2})$, respectively. In following sections we examine the probability that the receiver erroneously decides in favor of $\boldsymbol{S}_{1}$ when $\boldsymbol{S}_{0}$ was actually transmitted (i.e., $\mathrm{P}\left(\boldsymbol{S}_{0} \rightarrow\right.$ $\left.\boldsymbol{S}_{1}\right)$ ) for various spatial scenarios. Note that in this case $\beta_{0,1}=2$.

\section{A. Effect of Antenna Spacing}

First we consider the effect of antenna spacing on the exact-PEP when the scattering environment is uncorrelated, i.e., $\boldsymbol{F}_{T}=\boldsymbol{I}_{2 M_{T}+1}$ and $\boldsymbol{F}_{R}=\boldsymbol{I}_{2 M_{R}+1}$. Consider a system with two transmit antennas and two receive antennas, where the two transmit antennas are placed in a circular aperture of radius $0.25 \lambda$ (antenna separation ${ }^{4}=0.5 \lambda$ ) and the two receive antennas are placed in a circular aperture of radius $r$ (antenna separation $=2 r$ ). Fig. 1 shows the exact pairwise error probability performance of the DSTC for the error event $\boldsymbol{S}_{0} \rightarrow \boldsymbol{S}_{1}$ and receive antenna separations $0.1 \lambda, 0.2 \lambda, 0.5 \lambda$ and $\lambda$. Also shown in Fig.1 for comparison is the exact-PEP (27) for the i.i.d. slow fading channel corresponding to the error event $\boldsymbol{S}_{0} \rightarrow \boldsymbol{S}_{1}$.

As we can see from the figure, the effect of antenna separation on the exact-PEP is not significant when the receive antenna separation is $0.5 \lambda$ or higher. However, the effect is significant when the receive antenna separation is small. For example, at PEP $10^{-4}$, the realistic PEPs are about $1 \mathrm{~dB}$ and $3 \mathrm{~dB}$ away from the i.i.d. chanel performance results for $0.2 \lambda$ and $0.1 \lambda$ receive antenna separations, respectively. From these observations, we can emphasize that the effect of antenna spacing on the performance of DSTC is minimum for higher antenna separations whereas the effect is significant for smaller antenna separations.

\footnotetext{
${ }^{4}$ In a 3-D isotropic scattering environment, antenna separation $0.5 \lambda$ (first null of the order zero spherical Bessel function) gives zero spatial correlation, but here we constraint our analysis to a 2-D scattering environment. The spatial correlation function in a 2-D isotropic scattering environment is given by a Bessel function of the first kind. Therefore, antenna separation $\lambda / 2$ does not give zero spatial correlation in a 2-D isotropic scattering environment.
} 


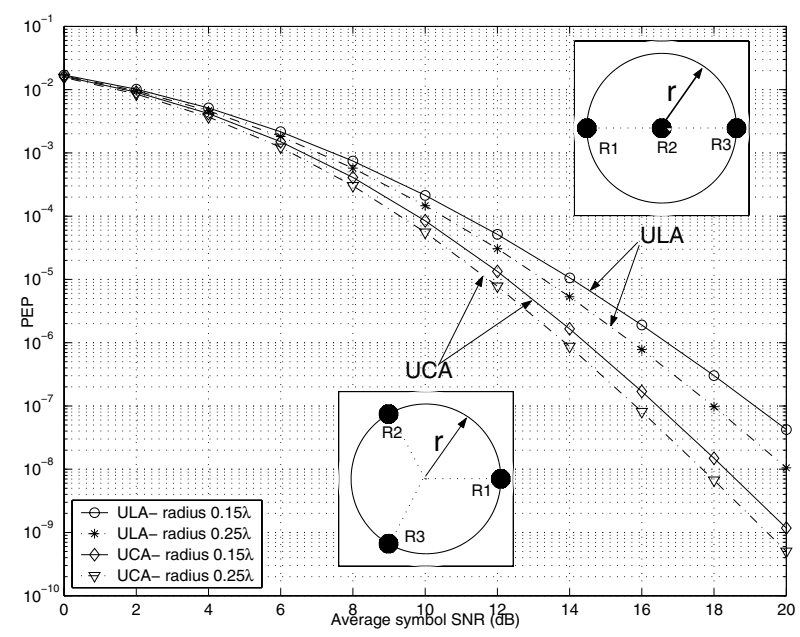

Fig. 2. Exact-PEP performance of DSTC scheme with two transmit and three receive antennas for UCA and ULA receiver antenna configurations; $\beta_{0,1}=2$.

\section{B. Effect of Antenna Configuration}

In this section, we compare the PEP performance of the DSTC used in the previous section for different antenna configurations at the receiver antenna array. For example, we choose UCA and ULA antenna configurations ${ }^{5}$. Consider a system with two transmit antennas and three receive antennas. The two transmit antennas are placed half wavelength $(\lambda / 2)$ distance apart and the three receive antennas are placed within a fixed circular aperture of radius $r(=0.15 \lambda, 0.25 \lambda)$, as shown in Fig.2. The exact-PEP performance for the error event $\boldsymbol{S}_{0} \rightarrow \boldsymbol{S}_{1}$, corresponding to $\beta_{0,1}$, is also plotted in Fig 2 .

From Fig.2, it is observed that at high SNRs the performance given by the UCA antenna configuration outperforms that of the ULA antenna configuration. For example, at PEP $10^{-6}$, the performance differences between UCA and ULA are about $2.5 \mathrm{~dB}$ with $0.15 \lambda$ receiver aperture radius and about $2 \mathrm{~dB}$ with $0.25 \lambda$ receiver aperture radius. Therefore, as we illustrated here, one can use the PEP expression (28) to determine the best antenna placement within a given region which gives the maximum performance gain available from a DSTC scheme. Furthermore, the slope of the performance curve on a log scale corresponds to the diversity advantage of the code and the horizontal shift in the performance curve corresponds to the coding advantage. From Fig.2, we observed that as the radius of the receiver aperture decreases the diversity advantage of the code is reduced, particularly for the ULA antenna configuration. Here, the loss of diversity advantage is mainly due to the loss of rank of $\boldsymbol{J}_{R}$.

\section{Effect of Modal Correlation}

For simplicity, here we only consider the modal correlation ${ }^{6}$ effects at the receiver region and assume that the effective communication modes available at the transmitter region are uncorrelated, i.e., $\boldsymbol{F}_{T}=\boldsymbol{I}_{2 M_{T}+1}$. In [13], it was shown that the correlation between the $n$-th and $n^{\prime}$-th modes at the

\footnotetext{
${ }^{5}$ The exact-PEP expression we derived in this work can be applied to any arbitrary antenna configuration.

${ }^{6}$ Second order statistics of the scattering channel $\boldsymbol{H}_{S}$.
}

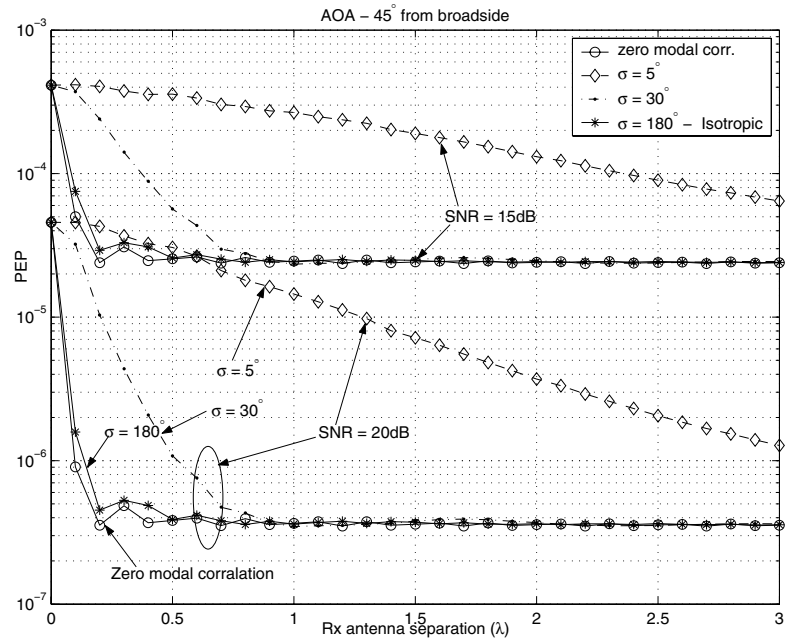

Fig. 3. Exact-PEP performance of the DSTC scheme with two transmit and two receive antennas against the receive antenna separation for a uniform limited power distribution at the receiver with mean angle of arrival $\phi_{0}=45^{\circ}$ from broadside and angular spreads $\sigma_{s}=\left[5^{\circ}, 30^{\circ}, 180^{\circ}\right]$; Transmit antenna antenna separation $0.5 \lambda$ and $\beta_{0,1}=2$.

receiver region due to the $m$-th mode at the transmitter region can be written as

$$
\gamma_{n, n^{\prime}}^{R x}=\int \mathcal{P}_{R x}(\phi) e^{-i\left(n-n^{\prime}\right) \phi} d \phi,
$$

where $\mathcal{P}_{R x}(\phi)=\int G(\varphi, \phi) d \varphi$ is the normalized azimuth power distribution of the scatterers surrounding the receiver antenna region. Note that the $\left(n, n^{\prime}\right)$-th element of $\boldsymbol{F}_{R}$ is given by (30) and $\boldsymbol{F}_{R}$ is a $\left(2 m_{R}+1\right) \times\left(2 m_{R}+1\right)$ matrix. Also note that $\mathcal{P}_{R x}(\phi)$ can be modeled using all common azimuth power distributions such as Uniform, Gaussian, Laplacian, Von-Mises, Polynomial, etc. Here we restrict our investigation only to the case of energy arriving uniformly over a limited angular spread $\sigma_{s}$ around a mean AOA $\phi_{0}$ (uniform limited azimuth power distribution). In this case, the modal correlation coefficient $\gamma_{n, n^{\prime}}^{R x}$ in the receiver region is given by

$$
\gamma_{n, n^{\prime}}^{R x}=\operatorname{sinc}\left(\left(n-n^{\prime}\right) \sigma_{s}\right) e^{-i\left(n-n^{\prime}\right) \phi_{0}} .
$$

Continuing the performance analysis, we now investigate the modal correlation effects on the performance of the rate-1 $2 \times 2$ DSTC scheme considered in previous sections. Consider a system with two transmit and two receive antennas where the two transmit antennas are placed $0.5 \lambda$ distance apart.

Fig. 3 shows the exact-PEP results for the error event $\boldsymbol{S}_{0} \rightarrow \boldsymbol{S}_{1}$ against the receiver antenna separation for a mean AOA $\phi_{0}=45^{\circ}$ from broadside ${ }^{7}$ and angular spreads $\sigma_{s}=$ $\left[5^{\circ}, 30^{\circ}, 180^{\circ}\right]$. Note that $\sigma_{s}=180^{\circ}$ represents the isotropic scattering environment. Since the exact-PEP expression we derived in this paper is valid only at high SNRs, the PEP results are plotted for $15 \mathrm{~dB}$ and $20 \mathrm{~dB}$ SNRs.

From Fig. 3 it is observed that for a given SNR, the performance of the DSTC scheme is improved as the receive antenna separation and the angular spread are increased. However, the performance does not improve monotonically with the increase in receive antenna separation. We also observed that when the

\footnotetext{
${ }^{7}$ Broadside angle is defined as the angle perpendicular to the line connecting the two antennas.
} 


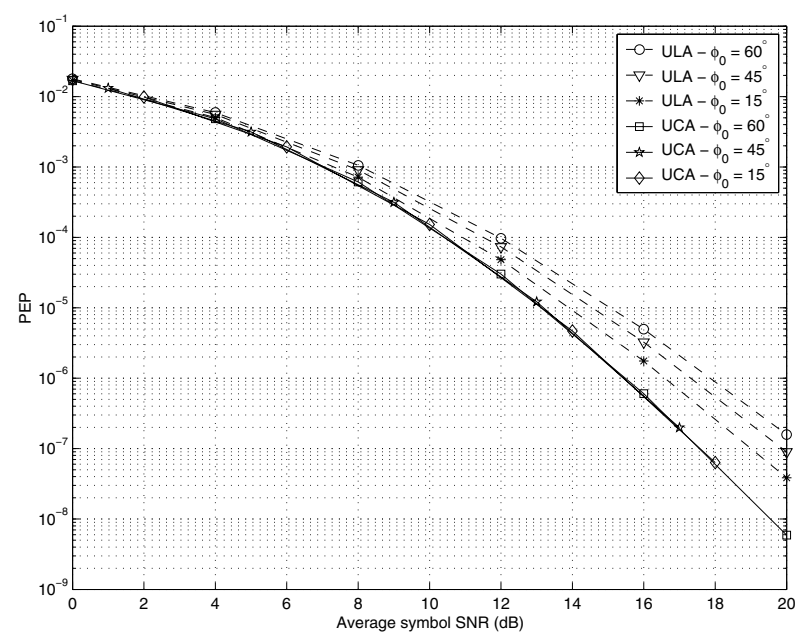

Fig. 4. Exact-PEP performance of DSTC scheme with two transmit and three receive antennas for UCA and ULA receiver antenna configurations for a uniform limited power distribution at the receiver with mean angle of arrivals $\phi_{0}=\left[60^{\circ}, 45^{\circ}, 15^{\circ}\right]$ from broadside and angular spread $\sigma_{s}=180^{\circ}$; Transmit antenna antenna separation $0.5 \lambda$, receive antenna separation $0.15 \lambda$ and $\beta_{0,1}=2$.

angular spread is quite small (e.g. $5^{\circ}$ ), we need to place the two receive antenna elements at least several wavelengths apart in order to achieve the maximum performance gain given by the space-time code. In this case, the loss of performance is mainly due to the higher concentration of energy closer to the mean AOA for small angular spreads.

Fig. 4 illustrates the effects of mean AOA on the exact PEP of DSTC for UCA and ULA antenna configurations at the receiver. Antenna elements at the receiver are placed within a fixed circular aperture of radius $0.15 \lambda$, similar to antenna configuration setup shown in Fig. 2 and the two transmit antennas are placed $0.5 \lambda$ distance apart. As before, we consider a uniform limited azimuth power distribution at the receiver with mean AOAs $\phi_{0}=\left[60^{\circ}, 45^{\circ}, 15^{\circ}\right]$ from broadside and angular spread $\sigma_{s}=180^{\circ}$.

From Fig. 4 we observed that the performance loss of the DSTC scheme is most pronounced for the ULA antenna configuration when the mean AOA is closer to $90^{\circ}$ (inline with the array). But, for the UCA antenna configuration, the performance loss is insignificant as the mean AOA moves away from broadside. This suggests that the UCA antenna configuration is less sensitive to change of mean AOA compared to the ULA antenna configuration. Hence, the UCA antenna configuration is best suited to employ a space-time code.

Using the results we obtained thus far, we can claim that, in general, differential space-time codes are susceptible to spatial fading correlation effects, in particular, when the antenna separation and the angular spread are small.

\section{Conclusion}

Using an MGF-based approach, we have derived an analytical expression for the exact pairwise error probability of a differentially space-time coded system operating over a spatially correlated slow fading channel. This analytical PEP expression fully accounts for antenna separation, antenna geometry and surrounding azimuth power distributions, both at the receiver and the transmitter antenna arrays. In practice, this expression can be used as a tool to estimate or predict the error performance of a differential space-time coded system under a given antenna configuration and surrounding azimuth power distribution parameters.

\section{REFERENCES}

[1] I. E. Telatar, "Capacity of multi-antenna gaussian channels," Tech. Repo., AT\&T Bell Labs, 1995.

[2] G. J. Foschini and M. J. Gans, "On limits of wireless communications in a fading environment when using multiple antennas," Wireless Personal Communications, vol. 6, pp. 311-335, 1998.

[3] V. Tarokh, N. Seshadri, and A. R. Calderbank, "Space-time codes for high data rate wireless communication: performance criterion and code construction," IEEE Trans. Info. Theory, vol. 44, no. 1, pp. 744-765, Mar. 1998.

[4] S. Alamouti, "A simple transmit diversity technique for wireless communications," IEEE Trans. Commun., vol. 16, no. 8, pp. 1451-1458, Oct. 1998.

[5] V. Tarokh, H. Jafarkhani, and A. R. Calderbank, "Space-time codes from orthogonal designs," IEEE Trans. Info. Theory, vol. 45, no. 5, pp. 1456-1467, July 1999.

[6] V. Tarokh and H. Jafarkhani, "A differential detection scheme for transmit diversity," IEEE Journal on Selected Areas in Communications, vol. 18, no. 7, pp. 1169-1174, July 2000.

[7] B. L. Hughes, "Differential space-time modulation," IEEE Trans. Info. Theory, vol. 46, pp. 2567-2578, Nov. 2000.

[8] B. M. Hochwald and W. Sweldens, "Differential unitary space-time modulation," IEEE Trans. Commun., vol. 48, no. 12, pp. 2041-2052, Dec. 2000.

[9] C. Gao, A. M. Haimovich, and D. Lao, "Bit error probability for spacetime block code with coherent and differential detection," in IEEE 56th Vehicular Technology Conference (VTC 2002-Fall), Vancouver, Canada, Sept. 2002, vol. 1, pp. 410-414.

[10] Z. Bagley and C. Schlegel, "Pair-wise error probability for space-time codes under coherent and differentailly coherent decoding," submitted to IEEE Trans. Commun.

[11] V. K. Nguyen, "Differential encoding technique for multi-antenna systems with correlated rayleigh fading channels," in IEEE International Conf. on Industrial Tech. (to appear), Hong Kong, Dec. 2005.

[12] M. K. Simon, "Evaluation of average bit error probability for space-time coding based on a simpler exact evaluation of pairwise error probability," International Journal on Communications and Networks, vol. 3, no. 3, pp. 257-264, Sept. 2001.

[13] T. A. Lamahewa, M. K. Simon, R. A. Kennedy, and T. D. Abhayapala, "Performance analysis of space-time codes in realistic propagation environments: A moment generating function-based approach," Journal Of Communication and Networks, vol. 7, no. 4, pp. 450-461, Dec. 2005.

[14] T. D. Abhayapala, T. S. Pollock, and R. A. Kennedy, "Spatial decomposition of MIMO wireless channels," in The Seventh International Symposium on Signal Processing and its Applications, Paris, France, July 2003, vol. 1, pp. 309-312.

[15] H. M. Jones, R. A. Kennedy, and T. D. Abhayapala, "On dimensionality of multipath fields: Spatial extent and richness," in Proc. IEEE Int. Conf. Acoust., Speech, Signal Processing, ICASSP'2002, Orlando, Florida, May 2002, vol. 3, pp. 2837-2840.

[16] T. S. Pollock, "Correlation Modelling in MIMO Systems: When can we Kronecker?," in Proc. 5th Australian Communications Theory Workshop, Newcastle, Australia, Feb. 2004, pp. 149-153.

[17] M. K. Simon and M. S. Alouini, Digital Communications over Fading Channels, John Wiley \& Sons, Hoboken, New Jersey, USA, second edition, Nov. 2004.

[18] G. L. Turin, "The characteristic function of hermetian quadratic forms in complex normal random variables," Biometrika, vol. 47, no. 1/2, pp. 199-201, June 1960. 